Published in final edited form as:

J Surg Res. 2009 December ; 157(2): 243-250. doi:10.1016/j.jss.2008.10.025.

\title{
Development of a sleeve gastrectomy weight-loss model in obese Zucker rats
}

\author{
Peter P. Lopez, M.D., Susannah E. Nicholson, M.D., Gabriel E. Burkhardt, M.D., Robert A. \\ Johnson, Ph.D., and Fruzsina K. Johnson, M.D. \\ Divisions of General and Laparoendoscopic Surgery and Trauma and Emergency Surgery, \\ Department of Surgery, University of Texas Health Science Center at San Antonio, and the National \\ Trauma Institute (NTI), San Antonio, TX
}

\begin{abstract}
Background: Obesity promotes the development of diabetes and cardiovascular disease. The most effective weight-loss treatment is bariatric surgery, but results greatly vary depending on the procedure. Sleeve gastrectomy (SG) has recently emerged as a reduced risk weight-loss procedure for super obese patients. However, the mechanism of weight-loss from SG and its effects on obesityinduced complications are yet to be determined. Our goal was to develop an experimental model of SG in genetically obese rats.
\end{abstract}

Materials and Methods: Male obese Zucker rats (400-500g, leptin-insensitive) were anesthetized with isoflurane. After a midline laparotomy, the stomach was clamped, the greater curvature was excised and a triple suture line was used to close the gastric remnant. Sham rats underwent laparotomy only. Metabolic parameters were followed for 14 days after surgery.

Results: Caloric intake and body weight decreased in SG rats over 14 days by $98 \pm 10 \mathrm{kcal} / \mathrm{day}$ and $74 \pm 14 \mathrm{~g}$, respectively. Blood total cholesterol levels were lower in rats that lost weight. Furthermore, blood glucose levels were lower in rats that lost weight. Active ghrelin levels were unchanged in SG rats 14 days after surgery.

Conclusions: These results show that SG promotes weight-loss in obese Zucker rats. Furthermore, SG-induced weight-loss is accompanied by improved plasma cholesterol and glucose profile.

However, SG does not promote a prolonged decrease in ghrelin levels. These results suggest that SG is an effective weight-loss procedure in leptin-insensitivity to improve the lipid profile and decrease insulin resistance and these effects might be independent of changes in ghrelin levels.

\section{Keywords}

gastric partitioning; sleeve gastrectomy; obesity; body weight; insulin resistance; metabolic indices; ghrelin

(C) 2009 Elsevier Inc. All rights reserved.

Corresponding author: Fruzsina K. Johnson, M.D. Division of Trauma and Emergency Surgery Department of Surgery University of Texas Health Science Center at San Antonio 7703 Floyd Curl Drive MC 7740 San Antonio, TX 78229 Tel.: (210) 567-0583 Fax: (210) 567-0003 johnsonf@uthscsa.edu.

Publisher's Disclaimer: This is a PDF file of an unedited manuscript that has been accepted for publication. As a service to our customers we are providing this early version of the manuscript. The manuscript will undergo copyediting, typesetting, and review of the resulting proof before it is published in its final citable form. Please note that during the production process errors may be discovered which could affect the content, and all legal disclaimers that apply to the journal pertain. 


\section{Introduction}

The obesity epidemic represents an emerging public health crisis (1) because obesity promotes the development of various diseases, such as cardiovascular disease and type 2 diabetes, that greatly increase mortality (2). Insulin resistance is a key metabolic feature that often precedes the development of type 2 diabetes. Body weight reduction improves metabolic status and decreases the risk of diabetes $(1,2)$, but weight control is difficult for most patients. Bariatric surgical procedures are currently the most effective approach to achieve long-term weight-loss $(3,4)$ and are traditionally characterized by the primary mechanism of weight-loss, namely intestinal malabsorption or gastric restriction (5). Procedures that combine gastric restriction and malabsorption, such as Roux-en-Y bypass (RYGB), promote superior and longer lasting weight-loss and elimination of co-morbidities compared to purely restrictive maneuvers (6, 7). Furthermore, RYGB promotes complex metabolic changes which promote weight-loss (5) and make it a preferred procedure for bariatric surgery (8). However, RYGB is an invasive procedure with multiple anastomoses which makes it less suitable for high-risk patients. For example, super-obese patients (Body Mass Index - BMI $>60 \mathrm{~kg} / \mathrm{m}^{2}$ ) would benefit the most from RYGB, but they represent the greatest surgical risk (9) which often limits their treatment options.

Sleeve gastrectomy, an advancement of the Magenstrasse and Mill technique $(10,11)$, has been introduced as the initial risk reduction procedure as part of a two-stage restrictivemalabsorptive RYGB maneuver in super-obese patients (12). During sleeve gastrectomy the gastric volume is restricted and the fundus is separated from the rest of the stomach. Recently, sleeve gastrectomy was shown to lower levels of the appetite-stimulant gastric hormone, ghrelin and produce effective lasting weight loss (13). These studies raised the potential of using sleeve gastrectomy as a stand-alone procedure in high-risk super-obese patients (14, 15). However, the mechanism of weight-loss from sleeve gastrectomy or its effects on comorbidities, such as type 2 diabetes have not yet been identified. Given that the accelerating obesity epidemic continues to elevate the number of super-obese patients, and sleeve gastrectomy may represent an effective low-risk bariatric solution for these patients (16), these questions are important to answer.

While a recent study compared weight-loss and metabolic effects of sleeve gastrectomy in various rodent models of obesity; that study found that it only had a minimal and transient effect in genetically obese rats (17). As gastric sleeve surgery has been shown to be an effective weight-loss procedure in humans (13), our goal was to develop an experimental model of sleeve gastrectomy that successfully reduces weight in genetically obese rats to examine its shortterm metabolic effects. For this purpose, we adapted the advanced Magenstrasse and Mill procedure to male obese Zucker (fa/fa) rats, which bear an autosomal recessive mutation of the leptin receptor gene (18) that leads to chronic hyperphagia resulting in morbid obesity $(19,20)$. We followed food intake and body weight daily for 14 days, then measured blood glucose, cholesterol, ghrelin and insulin levels at the end of the study.

\section{Materials and Methods}

Animals

Male obese Zucker rats (fa/fa, $\mathrm{n}=23$ ) were used for the study. Animals were delivered at 9-10 weeks of age and allowed to acclimatize for a week before subjected to one of the surgical procedures described below. Age matched lean Zucker rats (Harlan, Indianapolis, IN) with sham surgery $(\mathrm{n}=5)$ were used as controls for the metabolic studies. Additional lean Zucker rats without sham or sleeve surgery $(n=8)$ were used as controls for the blood metabolic and hormone values. Rats were housed in a controlled environment in the Department of Laboratory Animal Resources and had free access to purified rodent diet (AIN 76-A, Dyets Inc., 
Bethlehem, PA) and tap water until the day before the surgical procedures. All procedures were approved by the University of Texas Health Science Center at San Antonio Institutional Animal Care and Use Committee.

\section{Sleeve Gastrectomy}

We adapted the commonly used modified Magenstrasse and Mill procedure $(10,11)$ from humans to rats ( $\mathrm{n}=12$ obese rats). After 4-6 hr food deprivation, obese rats were anesthetized with isoflurane (5\% for induction and 3\% for maintenance) administered through a rodent surgical mask using a veterinary anesthesia machine (VMR, Matrx) equipped with $100 \%$ oxygen tank. The abdomen was shaved using surgical clippers (Andis AG, Shelton, CT). A 4 $\mathrm{cm}$ midline epigastric incision was made, then the terminal esophagus and the lesser and greater curvature of the stomach were dissected free. The anatomy of the rat stomach is different from human. The rat esophagus enters a thin walled non-glandular forestomach, limited from a second thick walled glandular corpus compartment with a fold of tissue forming a limiting ridge (margo plicatus) (Figure 1A)(21,22). A tonsil clamp was placed along the greater curvature from the antrum to the fundus across the forestomach and glandular stomach. A scalpel was used to divide the greater curvature along the tonsil clamp removing approximately $90 \%$ of the forestomach and $70 \%$ of the glandular stomach (Figure 1A). The divided stomach was then closed with 3-0 silk suture (Ethicon, Piscataway, NJ) in three layers creating the gastric sleeve (Figure 1B). The first layer was a running u-stitch under the clamp, the clamp was then removed and a running locked stitch was placed over the u-stitch, followed with a third layer of a simple running stitch over the previous sutures. The wound was irrigated with $30 \mathrm{~mL}$ of normal saline. The abdominal wall was closed in two layers, the peritoneum and fascia were closed with 3-0 Vicryl suture (Ethicon, Piscataway, NJ) and the skin was closed with 3-0 Ethilon nylon suture (Ethicon, Piscataway, NJ) in a continuous fashion. Tissue adhesive (Vetbond, 3M, St. Paul, MN) was placed over the outer suture line to prevent the animals from opening their sutures post-operatively. Control rats underwent laparotomy, the stomach was elevated and replaced in position and the abdominal wall was closed as described above ( $\mathrm{n}=5$ lean sham and $\mathrm{n}=11$ obese sham rats). The rest of the lean animals $(\mathrm{n}=8)$ did not receive sleeve gastrectomy or sham surgery.

\section{Metabolic Study}

Rats were individually housed in rodent metabolism cages (Nalgene Nunc International, Rochester, NY) for two days prior and 14 days following the surgical procedure. Previous experience with Zucker rats suggested that laparotomies may lead to aberrations in caloric intake and weight gain that may span a week. Accordingly, we chose to double that period in our current study to ensure that the animals have stabilized. Body weights, food and water intakes, and urinary outputs were measured daily. Rats had free access to purified rodent diet (AIN 76-A) and tap water for two days. Surgery was performed on the third day after 4-6 hr food deprivation. The first two postoperative days, sleeve gastrectomy and sham animals had free access to a liquid nutritional supplement (Boost, Nestlé HealthCare Nutrition, Fremont, MI) administered through a second water bottle, and tap water. Starting the third postoperative day, animals had free access to purified rodent diet and tap water though the remainder of the study. Caloric intake was calculated based on the published nutritional content of the AIN 76A rodent diet $(3.9 \mathrm{kcal} / \mathrm{g})$ and Boost $(1 \mathrm{kcal} / \mathrm{mL}, 0.95 \mathrm{kcal} / \mathrm{g})$ multiplied by the consumption (g/day) and expressed as $\mathrm{kcal} / \mathrm{day}$.

\section{Blood Metabolic and Hormone Measurements}

In order to avoid the stressful effects of repeated blood drawings in these small animals, blood glucose, lipids, insulin and ghrelin levels were only measured at the end of the study. Obese rats, 14 days after surgery, and age-matched lean rats were anesthetized with isoflurane, and 
an indwelling carotid arterial catheter was implanted for blood sample collections. Blood samples were drawn for immediate determination of non-fasting blood glucose and total cholesterol (CardioChek analyzer, Polymer Technology Systems, Inc., Indianapolis, IN). Additional blood was collected in test tubes containing EDTA and plasma was harvested by centrifugation. Plasma samples were aliquotted and stored at $-20^{\circ} \mathrm{C}$ until analyzed. Plasma insulin, acylated and un-acylated ghrelin levels were determined using commercially available assay kits (Cayman Chemical, Ann Arbor, MI). After blood drawing, animals were euthanized by anesthesia overdose, wide-breaching of the thorax and removal of the heart in accordance with the recommendations of the American Veterinary Medical Association Panel on Euthanasia (23).

\section{Statistics}

All data are expressed as mean \pm SEM. Metabolic measurements were analyzed by analysis of variance using a computer statistical package (Systat, Systat Software, Inc., San Jose, CA). When significant differences were observed, orthogonal contrasts were performed as a post hoc analysis (24). All other data were analyzed by t tests. An $\alpha<0.05$ was considered statistically significant.

\section{Results \\ Metabolic Study}

We performed sleeve gastrectomy on 12 obese rats. In four animals out of 12 the remnant forestomach dilated to its original size or larger (Figure 1C) and these animals followed a diverse metabolic course from rats with successful gastric sleeve. Namely, the weight-loss was minimal and transient and the decreases in plasma glucose levels were slight. We realized that the ratio of failed sleeves rapidly diminished with experience indicating that these animals were part of a learning curve problem. Therefore, we excluded these animals from the analysis and metabolic data are presented as lean $(n=5)$ and obese $(n=11)$ sham controls, and successful gastric sleeve animals ( $\mathrm{n}=8$ obese rats).

Figure 2 shows calculated caloric intake of lean and obese Zucker rats for 2 days prior and 14 days following sham surgery, or sleeve gastrectomy. Before surgery, caloric intake was not different between the two obese groups; both were higher when compared to the lean sham group. On the first postoperative day, caloric intake fell in the lean $(97 \pm 6 \mathrm{kcal} / \mathrm{day}$ to $54 \pm 7 \mathrm{kcal} /$ day) and obese $(135 \pm 5 \mathrm{kcal} /$ day to $55 \pm 10 \mathrm{kcal} /$ day $)$ sham groups, but decreased to a greater extent in the gastric sleeve group $(136 \pm 3 \mathrm{kcal} / \mathrm{day}$ to $3 \pm 1 \mathrm{kcal} / \mathrm{day})$. Two days after surgery the caloric intake of the lean and obese sham animals substantially recovered (lean sham: 60 $\pm 3 \mathrm{kcal} /$ day, obese sham: $109 \pm 7 \mathrm{kcal} /$ day) and was stabilized throughout the remainder of the study. In rats with the gastric sleeves caloric intake remained low for 6 days and then increased slowly to $38 \pm 8 \mathrm{kcal} /$ day during the second week.

Figure 3 shows body weight of of lean and obese Zucker rats for 2 days prior and 14 days following sham surgery, or sleeve gastrectomy. Before surgery, body weight was not different in the obese sham and gastric sleeve groups (sham: $462 \pm 10 \mathrm{~g}$ vs. sleeve: $452 \pm 8 \mathrm{~g}$ ); both were higher compared to the lean sham group $(335 \pm 4 \mathrm{~g})$. In lean and obese sham animals body weight continued to rise after surgery and increased to $366 \pm 5 \mathrm{~g}$ (lean) and $525 \pm 7 \mathrm{~g}$ (obese) in two weeks. In rats with gastric sleeves, body weight fell immediately following surgery $(428 \pm 9 \mathrm{~g})$ and continued to decrease to $378 \pm 19 \mathrm{~g}$ in two weeks.

\section{Blood Metabolic and Hormone Measurements}

Figure 4 shows non-fasting blood glucose levels in lean rats, and obese rats with sham or gastric sleeve surgery. Obese rats with sham surgery have elevated blood glucose levels compared to 
lean rats (lean: $140 \pm 12 \mathrm{mg} / \mathrm{dL}$ vs. obese sham: $246 \pm 23 \mathrm{mg} / \mathrm{dL}$ ). Blood glucose levels were decreased in obese rats with sleeve gastrectomies $(153 \pm 15 \mathrm{mg} / \mathrm{dL})$ compared to obese sham rats.

Figure 5 shows total cholesterol levels in lean rats, and obese rats with sham or gastric sleeve surgery. Similar to blood glucose levels, obese rats with sham surgery have elevated total cholesterol levels compared to lean rats (lean: $100 \pm 0 \mathrm{mg} / \mathrm{dL}$ vs. obese sham: $214 \pm 23 \mathrm{mg}$ / $\mathrm{dL}$; lower limit of measurement is $100 \mathrm{mg} / \mathrm{dL}$ ). Total cholesterol levels were decreased in obese rats with sleeve gastrectomy $(107 \pm 4 \mathrm{mg} / \mathrm{dL})$.

Figure 6 shows triglyceride levels in lean rats, and obese rats with sham or gastric sleeve surgery. Triglyceride levels were not different between obese rats with sham surgery and lean rats (lean: $156 \pm 14 \mathrm{mg} / \mathrm{dL}$ vs. obese sham: $118 \pm 20 \mathrm{mg} / \mathrm{dL}$ ) and were not altered in obese rats with sleeve gastrectomy $(83 \pm 12 \mathrm{mg} / \mathrm{dL})$.

Figure 7 shows non-fasting insulin levels in lean rats, and obese rats with sham or gastric sleeve surgery. Obese rats with sham surgery have elevated blood insulin levels compared to lean rats (lean: $1.93 \pm 0.11 \mathrm{ng} / \mathrm{mL}$ vs. obese sham: $4.85 \pm 0.43 \mathrm{ng} / \mathrm{mL}$ ). Insulin levels were not decreased in obese rats with sleeve gastrectomy $(4.56 \pm 0.59 \mathrm{ng} / \mathrm{mL})$.

Figure 8 compares active (acylated) and total (acylated + un-acylated) ghrelin levels, and the active/total ghrelin ratio in lean animals, and obese rats with sham or gastric sleeve surgery. Obese rats with sham surgery have lower ghrelin levels compared to lean rats (lean: total 317.68 $\pm 31.14 \mathrm{pg} / \mathrm{mL}$, acylated $5.00 \pm 1.48 \mathrm{pg} / \mathrm{mL}$, ratio $1.79 \pm 0.49 \%$ vs. obese sham: total 229.72 $\pm 23.54 \mathrm{pg} / \mathrm{mL}$, acylated $0.00 \pm 0.00 \mathrm{pg} / \mathrm{mL}$, ratio $0.00 \pm 0.00 \%$ ). Ghrelin levels were not significantly different in obese rats with sleeve gastrectomy (total $297.87 \pm 49.90 \mathrm{pg} / \mathrm{mL}$, acylated $1.20 \pm 0.63 \mathrm{pg} / \mathrm{mL}$, ratio $0.45 \pm 0.30 \%$ ).

\section{Discussion}

The current study shows that surgical formation of a gastric sleeve can suppress appetite and promote weight-loss with reduced blood glucose and cholesterol levels in the obese Zucker rat. Since the obese Zucker rat is a genetic model of metabolic syndrome (18-20), the experimental approach constitutes a novel model for examining the effects of gastric resection on the deleterious events which arise from severe obesity.

A recent report suggested that gastric sleeve surgery produces only a slight and transient decrease in caloric intake and body weight in obese Zucker rats (17). In the current study, we found that sleeve gastrectomy can produce a prolonged reduction in caloric intake and promote sustained weight-loss. While we can only speculate on the reasons for the different experimental results, a potential explanation might lie in the unique anatomy of the rat stomach. The rat may serve as a viable species for experimental gastric surgical manipulations, but the anatomy of the rat stomach differs from that of humans $(21,22)$ and accordingly requires special considerations. The rat esophagus enters a thin walled non-glandular forestomach, limited from a second thick walled glandular corpus compartment with a fold of tissue forming a limiting ridge (margo plicatus) (22). We have noted in our study that the failures of the gastric sleeves are accompanied by preferential dilation of the forestomach remnant. While the basis of this phenomenon is unclear, we believe that these experimental surgical failures originate from increased luminal pressures in the thin walled forestomach remnant. Whether driven by appetite, surgical or inflammatory restrictions, these pressures could increase wall tension to weaken the integrity of the thin remnant wall. In turn this should preferentially promote dilation of the forestomach (see Figure 1C) with respect to the thick walled corpus region. Consistent with this speculation, we found increased success with sleeve procedures with time when we learned to focus extra attention on minimizing the size of the forestomach compartment. We 
also learned that our early animals with failed gastric sleeves followed a diverse metabolic course from rats with successful gastric sleeve. Namely, the decrease in caloric intake, as well as the weight-loss was minimal and transient similar to the recently published study (17) suggesting that the dilation of gastric sleeve might be a potential explanation for their different results. Our experience with rat stomach surgery is not unique, as another research group performing RYGB in obese Zucker rats found that initially the stomach staple line broke down in a large number of animals (25) resulting in recovery of weight and caloric intake.

The obese Zucker rat is a genetic model of morbid obesity (19) that displays traits typical of metabolic syndrome, including insulin resistance (20). We find, that the gastric sleeve procedure reduces caloric intake, promotes sustained reduction in weight and lowers levels of blood glucose. The resulting improvement of blood glucose arises without changes in plasma insulin which is potentially suggestive -but not confirmatory- of improved insulin responsiveness. It also appears that two weeks is insufficient time to completely normalize insulin sensitivity, as insulin levels do not return to those of the leans during that period. Interestingly, the recent report on gastric sleeve surgery in obese Zucker rats (17) found that insulin levels did not decrease, but blood glucose levels were decreased even two weeks after surgery despite the only transient reduction in caloric intake and body weight. These results suggest that either transient reduction in caloric intake and body weight can have prolonged beneficial effects, or that other effects of sleeve gastrectomy, beside weight-loss, might improve glucose homeostasis. These possibilities might be addressed in additional comprehensive studies beyond the scope of this current report.

Obese Zucker rats often display profound hypertriglyceridemia $(20,26)$ and hypercholesterolemia and we expected the same in this series. In the present study, rats were provided a synthetic defined chow (Dyets). This defined base chow has substantial levels of carbohydrates that facilitate appetite in compromised animals and also represent less bulk than natural diets, but it also possesses markedly lower levels of fat when compared to other popular commercial rat chows (Teklad). The triglyceride levels we measured in the present study were much lower than in our previous experience using obese Zucker rats fed regular rat chow (Teklad) (26). While cholesterol levels were reduced by the gastric surgery, that surgical procedure had little effect on triglyceride levels. Given our previous findings in obese Zucker rats fed Teklad (higher fat) chow (26), we believe that the lower fat Dyets chow may have limited the degree of hyperlipidemia in this current series.

Leptin is an adipose tissue-derived hormone which acts on the hypothalamus to signal satiety (27). The obese Zucker rat is known to possess a leptin receptor deficiency which drives appetite (18). Like morbidly obese humans, these animals prematurely develop renal (20) and cardiac hypertrophy (28), hepatic steatosis $(29,30)$ and endothelial dysfunction $(26,31)$. Whether limited intake improves organ function is unclear (30), as these obese rats after 11 weeks may possess pathologies which are ultimately irreversible by any treatment. While sleeve gastrectomy may confer little or no improvement after prolonged uncontrolled eating in this strain, this surgery may potentially prevent the onset or at least slow the progression of these conditions. Even so, the ability of the gastric sleeve to reduce weight is not likely to arise from changes in the preexisting genetically deficient leptin system.

As a counterpart to leptin (32), ghrelin is a hormone -largely generated and acetylated in the gastric fundus- which can act on the hypothalamus to stimulate appetite $(33,34)$. Others have suggested that stretching of the stomach can inhibit active ghrelin release to produce satiety (35). This claim is supported by clinical reports that some gastric procedures can reduce the levels of ghrelin (36-38) to trigger satiety at lower levels of gastric intake. When compared to their lean counterparts, we find that the obese strain of Zucker rats have virtually undetectable levels of acetylated ghrelin. Given their ravenous appetite, it is not surprising that gastric 
stretching might reduce its release. If so, it is also likely that the preexisting leptin receptor deficiency is enough to drive the appetite even when active ghrelin is absent. These results are in agreement with the recent study in obese Zucker rats (17) and suggest that obesity in these animals is independent of ghrelin production. The gastric sleeve procedure reduced food intake, but did not significantly change active ghrelin levels although the levels tended to increase. Similar results were reflected in a recently published article (17) where gastric sleeve surgery actually increased ghrelin levels in obese Zucker rats. This increase in active ghrelin might be a result of the lower food intake and the resulting decreased stretching of the stomach. These results might be - in part -explained by the unique anatomy of the rat stomach, as numerous studies have suggested that not the forestomach, but the glandular fundus is the region where ghrelin is activated (37-39). These results might limit the viability of this animal model as a clinical correlate, as in humans gastric sleeve surgery has been shown to lower ghrelin levels (13). While the different anatomy of the rat stomach makes rats perhaps a less than perfect model for human gastric surgery, it is important to point out that these results suggest that sleeve gastrectomy can be an effective weight-loss procedure even without lowering ghrelin levels.

In summary, we developed a novel experimental model of sleeve gastrectomy in obese Zucker rats. We showed that successful sleeve gastrectomy decreases caloric intake and promotes sustained weight-loss in this genetic model of morbid obesity. We also found that successful sleeve gastrectomy improves glucose and lipid profiles in obese Zucker rats. These results suggest that sleeve gastrectomy can be used as an effective bariatric procedure to promote weight-loss even in animals with genetic leptin resistance.

\section{Acknowledgments}

This work was supported by Department of Surgery in-house funds (PPL), National Heart, Lung and Blood Institute grant R01 HL76187 (RAJ), and funding from the National Trauma Institute (FKJ, RAJ).

\section{References}

1. Kopelman PG. Obesity as a medical problem. Nature 2000;404:635-643. [PubMed: 10766250]

2. Eckel RH, Grundy SM, Zimmet PZ. The metabolic syndrome. Lancet 2005;365:1415-1428. [PubMed: 15836891]

3. Brolin RE. Bariatric surgery and long-term control of morbid obesity. JAMA 2002;288:2793-2796. [PubMed: 12472304]

4. Mun EC, Blackburn GL, Matthews JB. Current status of medical and surgical therapy for obesity. Gastroenterology 2001;120:669-681. [PubMed: 11179243]

5. Cummings DE, Overduin J, Shannon MH, et al. Hormonal mechanisms of weight loss and diabetes resolution after bariatric surgery. Surg Obes Relat Dis 2005;1:358-368. [PubMed: 16925248]

6. Buchwald H, Avidor Y, Braunwald E, et al. Bariatric surgery: a systematic review and meta-analysis. JAMA 2004;292:1724-1737. [PubMed: 15479938]

7. Howard L, Malone M, Michalek A, et al. Gastric Bypass and Vertical Banded Gastroplasty- a Prospective Randomized Comparison and 5-Year Follow-up. Obes Surg 1995;5:55-60. [PubMed: 10733794]

8. Must A, Spadano J, Coakley EH, et al. The disease burden associated with overweight and obesity. JAMA 1999;282:1523-1529. [PubMed: 10546691]

9. Ren CJ, Patterson E, Gagner M. Early results of laparoscopic biliopancreatic diversion with duodenal switch: a case series of 40 consecutive patients. Obes Surg 2000;10:514-523. discussion 524. [PubMed: 11175958]

10. Johnston D, Dachtler J, Sue-Ling HM, et al. The Magenstrasse and Mill operation for morbid obesity. Obes Surg 2003;13:10-16. [PubMed: 12630607]

11. Carmichael AR, Sue-Ling HM, Johnston D. Quality of life after the Magenstrasse and Mill procedure for morbid obesity. Obes Surg 2001;11:708-715. [PubMed: 11775568] 
12. Regan JP, Inabnet WB, Gagner M, et al. Early experience with two-stage laparoscopic Roux-en-Y gastric bypass as an alternative in the super-super obese patient. Obes Surg 2003;13:861-864. [PubMed: 14738671]

13. Langer FB, Reza Hoda MA, Bohdjalian A, et al. Sleeve gastrectomy and gastric banding: effects on plasma ghrelin levels. Obes Surg 2005;15:1024-1029. [PubMed: 16105401]

14. Milone L, Strong V, Gagner M. Laparoscopic sleeve gastrectomy is superior to endoscopic intragastric balloon as a first stage procedure for super-obese patients (BMI > or =50). Obes Surg 2005;15:612617. [PubMed: 15946449]

15. Almogy G, Crookes PF, Anthone GJ. Longitudinal gastrectomy as a treatment for the high-risk superobese patient. Obes Surg 2004;14:492-497. [PubMed: 15130224]

16. Deitel, M.; Crosby, RD.; Gagner, M. Obes Surg; The First International Consensus Summit for Sleeve Gastrectomy (SG); New York City. October 25-27, 2007; 2008.

17. Pereferrer FS, Gonzalez MH, Rovira AF, et al. Influence of sleeve gastrectomy on several experimental models of obesity: metabolic and hormonal implications. Obes Surg 2008;18:97-108. [PubMed: 18066699]

18. Ionescu E, Sauter JF, Jeanrenaud B. Abnormal oral glucose tolerance in genetically obese (fa/fa) rats. Am J Physiol 1985;248:E500-506. [PubMed: 3887938]

19. Zucker LM. Hereditary obesity in the rat associated with hyperlipemia. Ann N Y Acad Sci 1965;131:447-458. [PubMed: 5216982]

20. Kasiske BL, O'Donnell MP, Keane WF. The Zucker rat model of obesity, insulin resistance, hyperlipidemia, and renal injury. Hypertension 1992;19:I110-115. [PubMed: 1730447]

21. Greene, E. Anatomy of the rat. Hafner Pub. Co.; New York: 1968.

22. Robert A. Proposed terminology for the anatomy of the rat stomach. Gastroenterology 1971;60:344345. [PubMed: 5102323]

23. 1993 Report of the AVMA Panel on Euthanasia. J Am Vet Med Assoc 1993;202:229-249. [PubMed: 8428829]

24. Snedecor, GW.; Cochran, WG. Statistical methods. Iowa State University Press; Ames, Iowa: 1980.

25. Xu Y, Ohinata K, Meguid MM, et al. Gastric bypass model in the obese rat to study metabolic mechanisms of weight loss. J Surg Res 2002;107:56-63. [PubMed: 12384065]

26. Johnson FK, Johnson RA, Durante W, et al. Metabolic syndrome increases endogenous carbon monoxide production to promote hypertension and endothelial dysfunction in obese Zucker rats. Am J Physiol Regul Integr Comp Physiol 2006;290:R601-608. [PubMed: 16284090]

27. Valassi E, Scacchi M, Cavagnini F. Neuroendocrine control of food intake. Nutr Metab Cardiovasc Dis 2008;18:158-168. [PubMed: 18061414]

28. Paulson DJ, Tahiliani AG. Cardiovascular abnormalities associated with human and rodent obesity. Life Sci 1992;51:1557-1569. [PubMed: 1435063]

29. Chanussot F, Ulmer M, Ratanasavanh R, et al. Influence of diet composition on obesity, hyperlipemia and liver steatosis in Zucker fa/fa rats pair-fed with Zucker Fa/- rats. Int J Obes 1984;8:259-270. [PubMed: 6378817]

30. Kaminski DL, Mueller E, Jellinek M. Long-term effects of small intestinal bypass on hepatic lipid content in congenitally obese rats. Int J Obes 1984;8:21-30. [PubMed: 6706457]

31. Frisbee JC, Stepp DW. Impaired NO-dependent dilation of skeletal muscle arterioles in hypertensive diabetic obese Zucker rats. Am J Physiol Heart Circ Physiol 2001;281:H1304-1311. [PubMed: 11514301]

32. Cummings DE, Foster KE. Ghrelin-leptin tango in body-weight regulation. Gastroenterology 2003;124:1532-1535. [PubMed: 12730891]

33. Nakazato M, Murakami N, Date Y, et al. A role for ghrelin in the central regulation of feeding. Nature 2001;409:194-198. [PubMed: 11196643]

34. Ghigo E, Broglio F, Arvat E, et al. Ghrelin: more than a natural GH secretagogue and/or an orexigenic factor. Clin Endocrinol (Oxf) 2005;62:1-17. [PubMed: 15638864]

35. Cummings DE, Shannon MH. Roles for ghrelin in the regulation of appetite and body weight. Arch Surg 2003;138:389-396. [PubMed: 12686525] 
36. Cummings DE, Weigle DS, Frayo RS, et al. Plasma ghrelin levels after diet-induced weight loss or gastric bypass surgery. N Engl J Med 2002;346:1623-1630. [PubMed: 12023994]

37. Fruhbeck G, Diez Caballero A, Gil MJ. Fundus functionality and ghrelin concentrations after bariatric surgery. N Engl J Med 2004;350:308-309. [PubMed: 14724316]

38. Fruhbeck G, Diez-Caballero A, Gil MJ, et al. The decrease in plasma ghrelin concentrations following bariatric surgery depends on the functional integrity of the fundus. Obes Surg 2004;14:606-612. [PubMed: 15186626]

39. Ariyasu H, Takaya K, Tagami T, et al. Stomach is a major source of circulating ghrelin, and feeding state determines plasma ghrelin-like immunoreactivity levels in humans. J Clin Endocrinol Metab 2001;86:4753-4758. [PubMed: 11600536] 

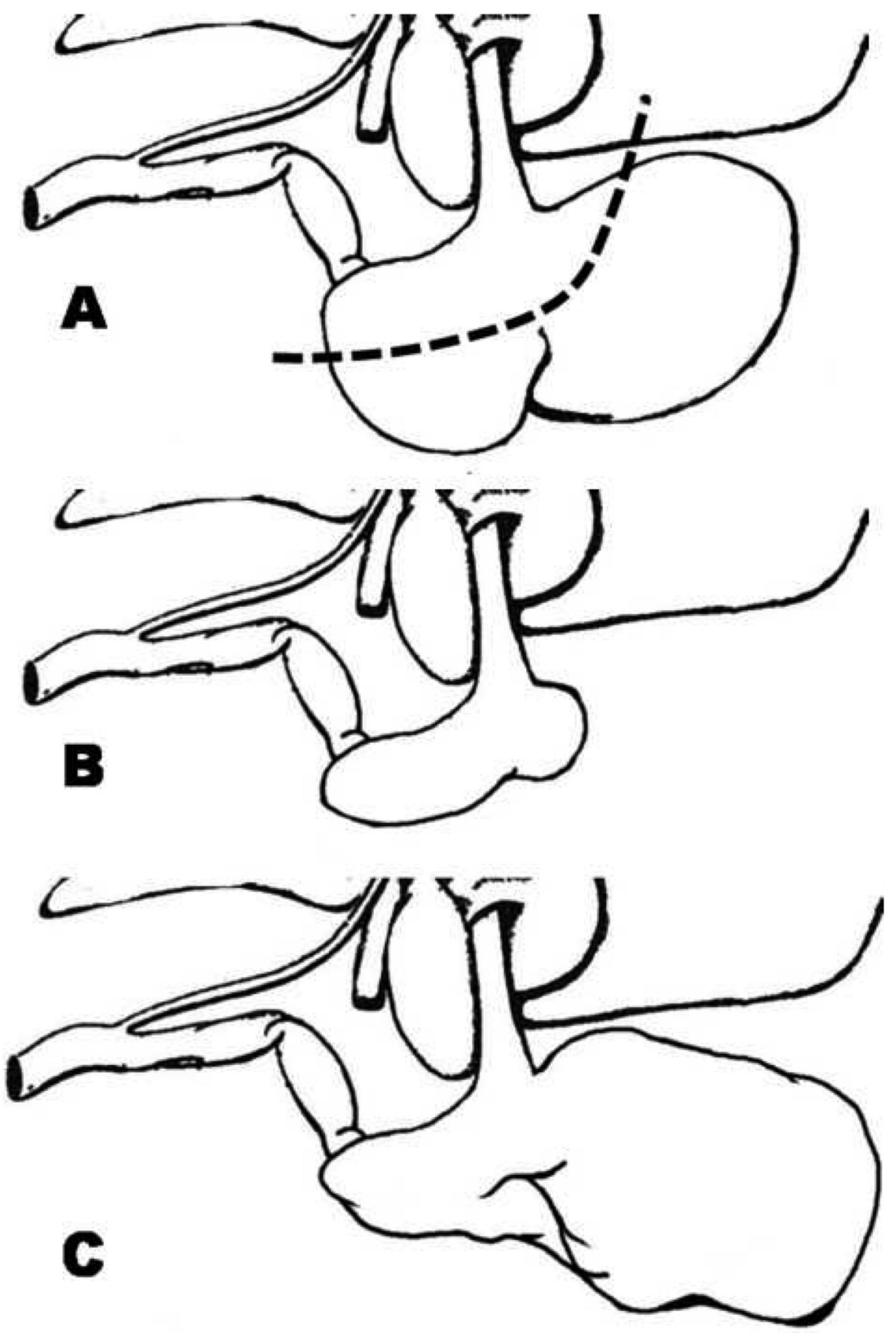

Figure 1.

Schematic illustrations of the rat stomach. A: Intact rat stomach. Dashed line represents the resection line. B: Rat stomach following sleeve gastrectomy. C: Rat stomach following failed gastric sleeve surgery with forestomach dilatation. 


\section{Caloric Intake}

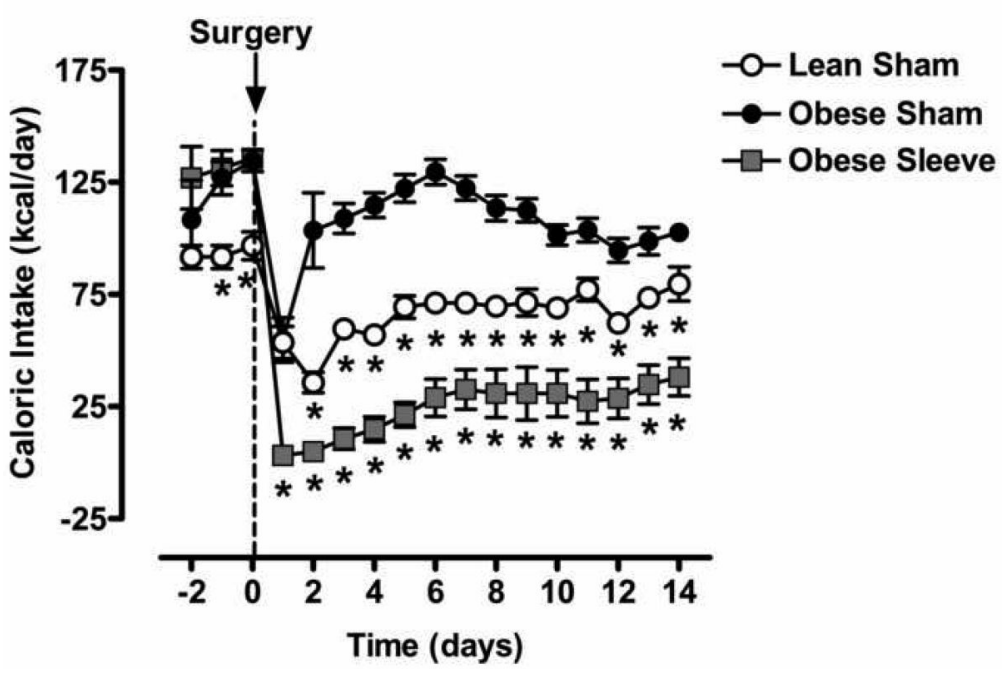

Figure 2.

Caloric intake of lean (open circles, $\mathrm{n}=5$ ) and obese Zucker rats for 2 days prior and 14 days following sham surgery (closed circles, $n=11$ ), or sleeve gastrectomy (grey squares, $n=8$ ). Data are expressed as mean \pm S.E.M. * represents $\mathrm{P}<0.05$ relative to the obese sham group. 


\section{Body Weight}

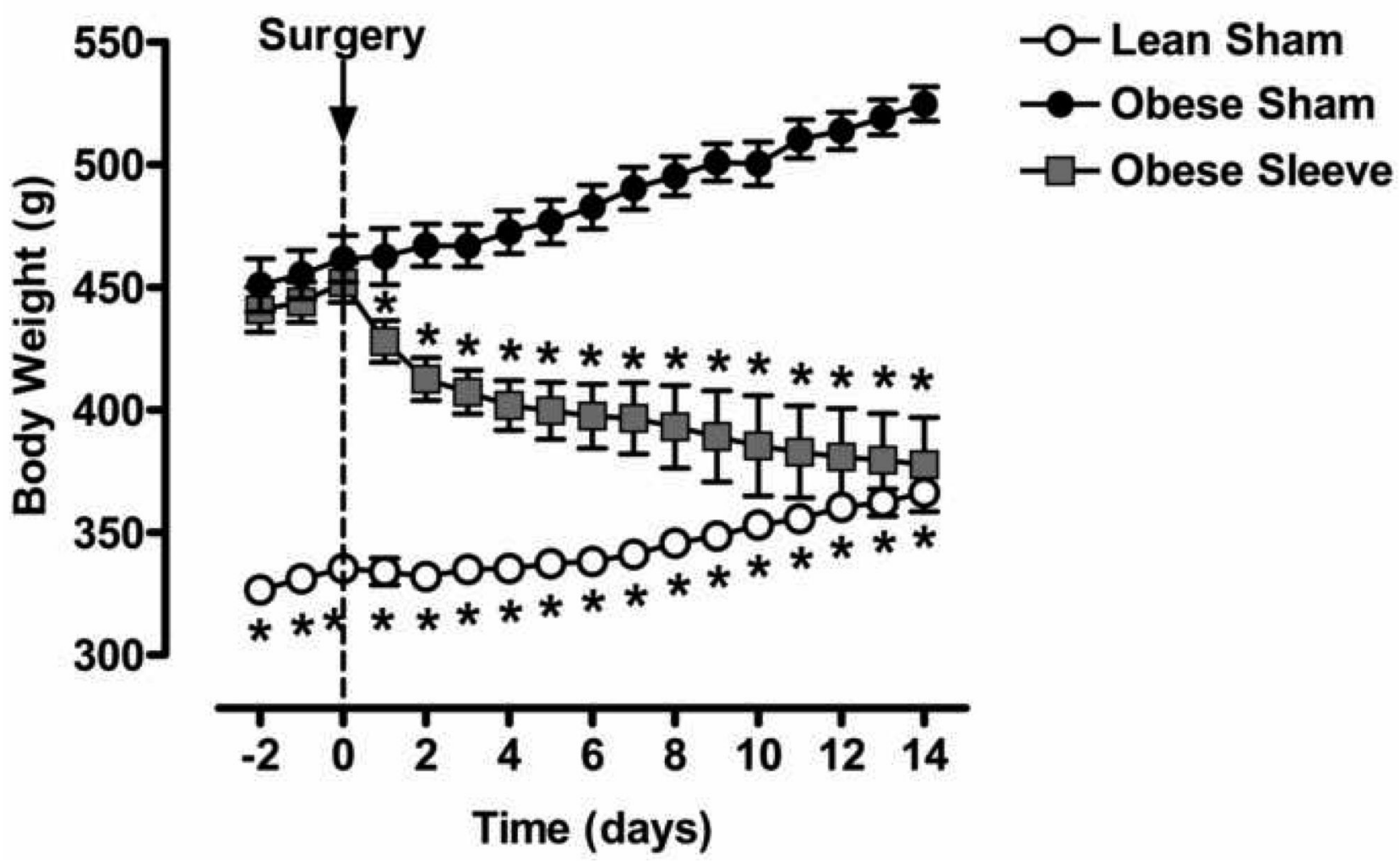

Figure 3.

Body weight of lean (open circles, $n=5$ ) and obese Zucker rats for 2 days prior and 14 days following sham surgery (closed circles, $n=11$ ), or sleeve gastrectomy (grey squares, $n=8$ ). Data are expressed as mean \pm S.E.M. * represents $\mathrm{P}<0.05$ relative to the obese sham group. 


\section{Glucose}

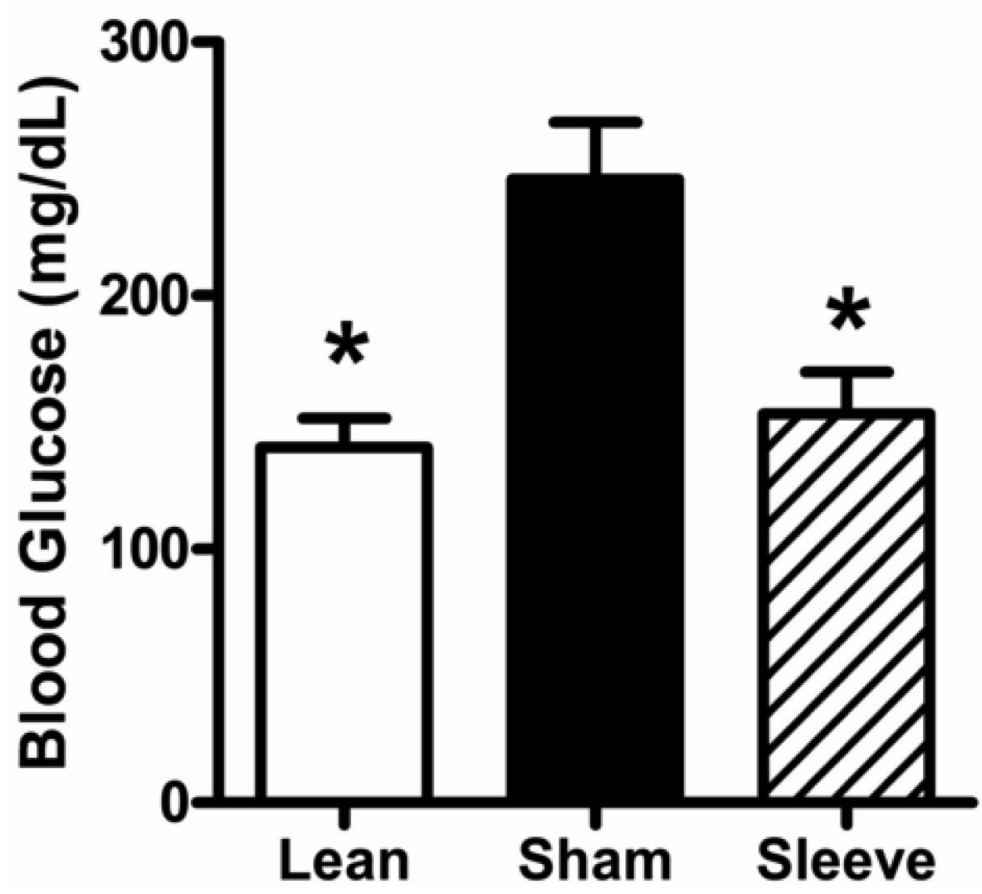

Figure 4.

Non-fasting blood glucose levels in lean rats (open bar, $n=8$ ), and obese Zucker rats 14 days following sham surgery (closed bar, $n=11$ ), or sleeve gastrectomy (hatched bar, $n=8$ ). Data are expressed as mean \pm S.E.M. * represents $\mathrm{P}<0.05$ relative to the obese sham group. 


\section{Cholesterol}

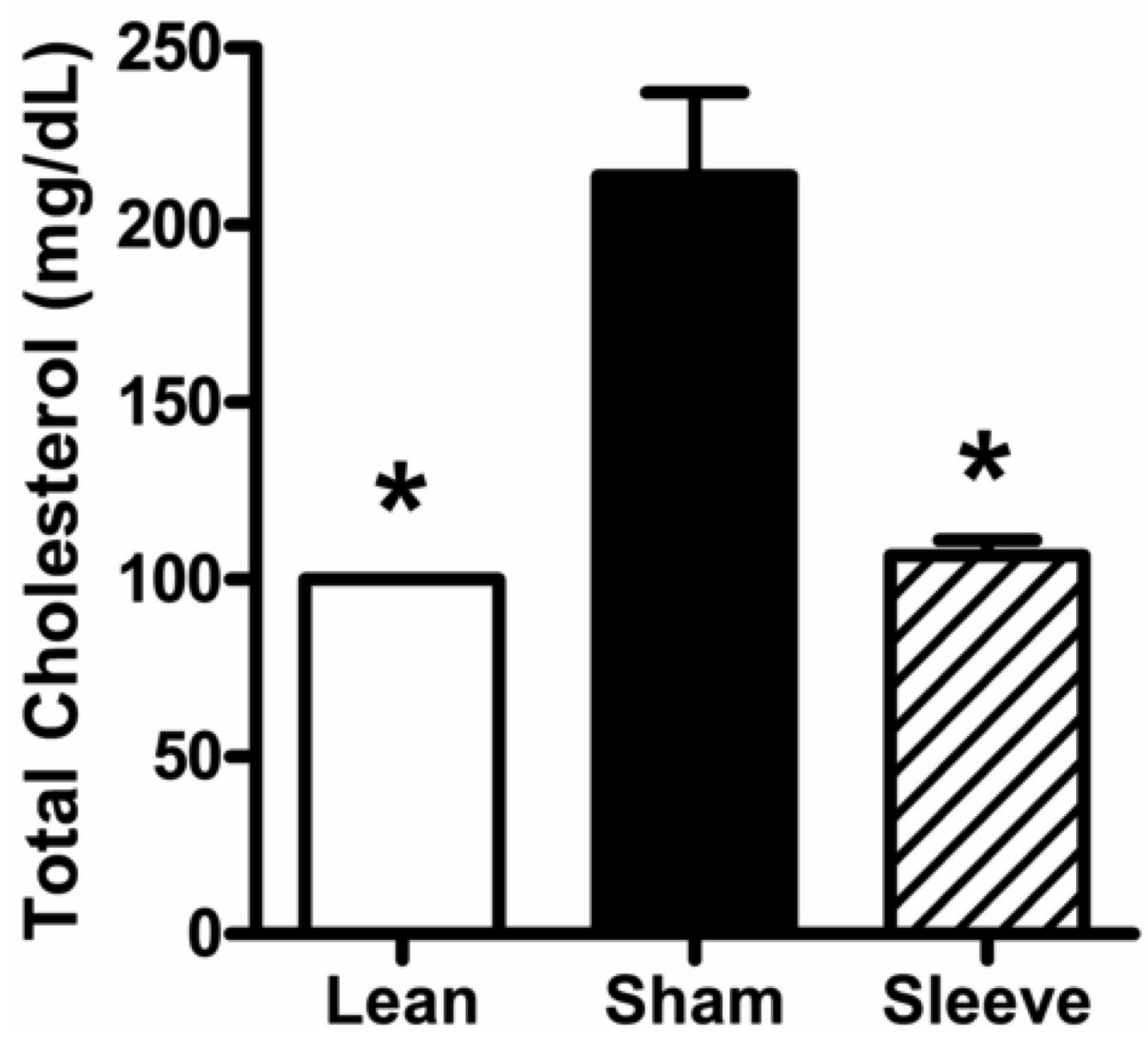

Figure 5.

Non-fasting total cholesterol levels in lean rats (open bar, $n=5$ ), and obese Zucker rats 14 days following sham surgery (closed bar, $n=11$ ), or sleeve gastrectomy (hatched bar, $n=8$ ). Data are expressed as mean \pm S.E.M. * represents $\mathrm{P}<0.05$ relative to the obese sham group. 


\section{Triglycerides}

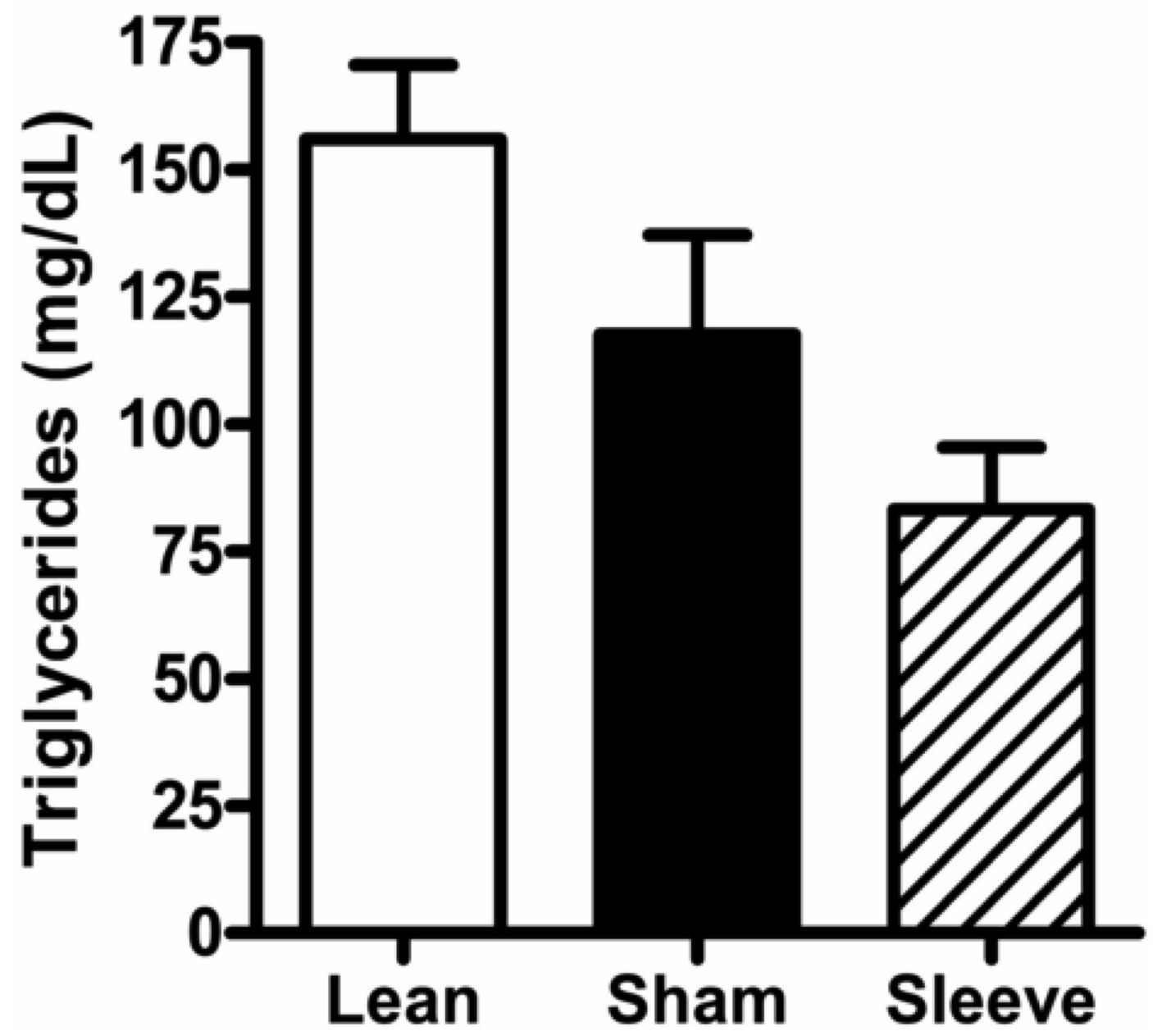

Figure 6.

Non-fasting triglyceride levels in lean rats (open bar, $n=5$ ), and obese Zucker rats 14 days following sham surgery (closed bar, $\mathrm{n}=11$ ) or sleeve gastrectomy (hatched bar, $\mathrm{n}=8$ ). Data are expressed as mean \pm S.E.M. 


\section{Insulin}

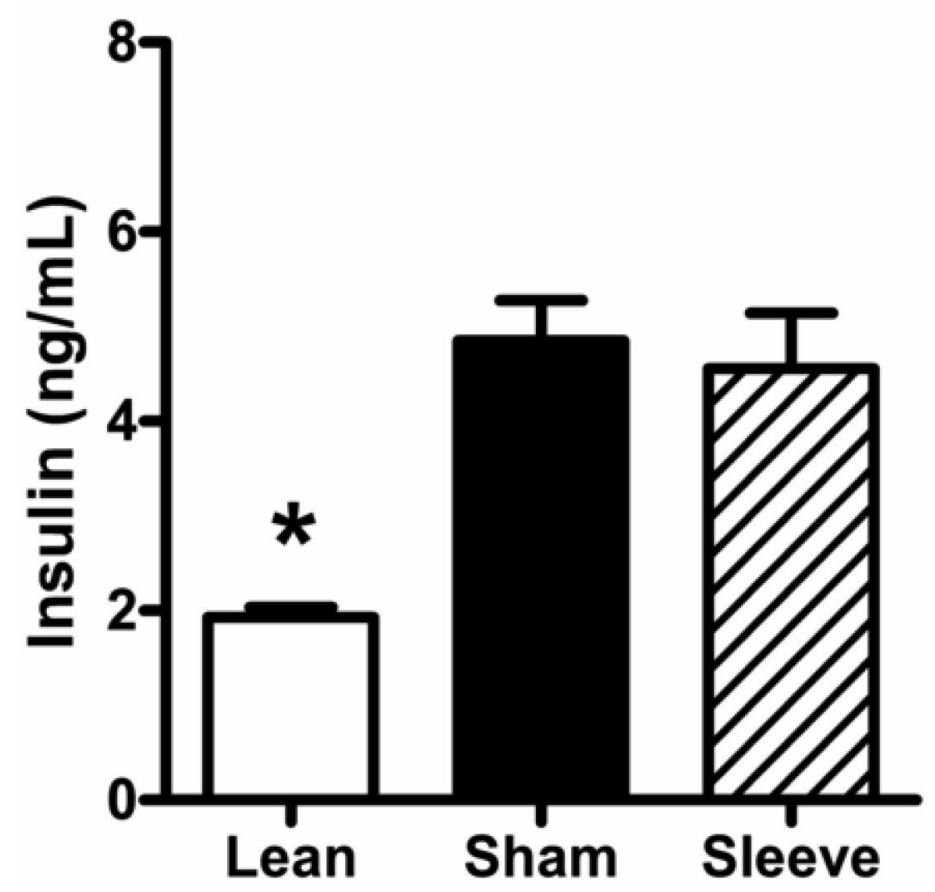

Figure 7.

Non-fasting insulin levels in lean rats (open bar, $n=5$ ), and obese Zucker rats 14 days following sham surgery (closed bar, $n=11$ ) or sleeve gastrectomy (hatched bar, $n=8$ ). Data are expressed as mean \pm S.E.M. * represents $\mathrm{P}<0.05$ relative to the obese sham group. 
Total Ghrelin

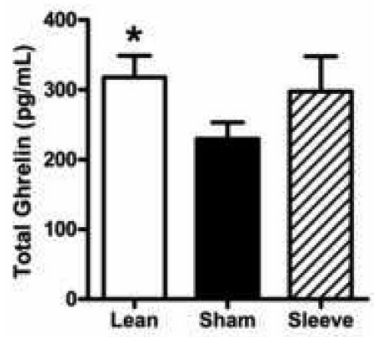

Active Ghrelin

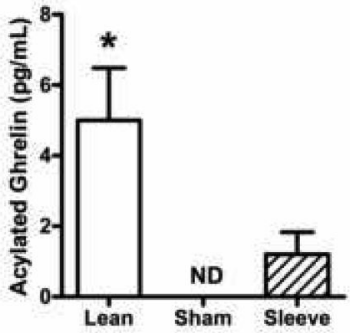

Active/Total Ghrelin Ratio

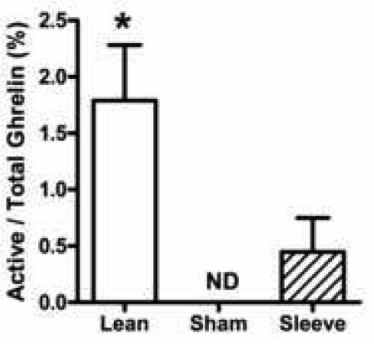

Figure 8.

Non-fasting ghrelin levels in lean rats (open bars, $n=5$ ), and obese Zucker rats 14 days following sham surgery (closed bars, $n=11$ ) or sleeve gastrectomy (hatched bars, $n=8$ ). A: Total ghrelin levels calculated as acylated ghrelin levels + un-acylated ghrelin levels. B: Acylated (active) ghrelin levels. C: Active / total ghrelin ratio. Data are expressed as mean \pm S.E.M. * represents $\mathrm{P}<0.05$ relative to the obese sham group. 